\title{
Technè
}

La science au service de l'histoire de l'art et de la préservation des biens culturels

44 | 2016

Archives de l'humanité : les restes humains patrimonialisés

\section{Un sujet inhabituel pour la revue Technè}

Introduction

Noëlle Timbart, Hélène Guichard et Alain Froment

\section{(2) OpenEdition}

Journals

Édition électronique

URL : http://journals.openedition.org/techne/904

DOI : $10.4000 /$ techne.904

ISSN : 2534-5168

Éditeur

C2RMF

Édition imprimée

Date de publication : 1 novembre 2016

Pagination : 4-7

ISBN : 978-2-7118-6339-6

ISSN : 1254-7867

Référence électronique

Noëlle Timbart, Hélène Guichard et Alain Froment, «Un sujet inhabituel pour la revue Technè », Technè [En ligne], 44 | 2016, mis en ligne le 19 décembre 2019, consulté le 23 septembre 2020. URL : http:// journals.openedition.org/techne/904 ; DOI : https://doi.org/10.4000/techne.904

\section{(c) (i) (2) $\Theta$}

La revue Technè. La science au service de l'histoire de l'art et de la préservation des biens culturels est mise à disposition selon les termes de la Licence Creative Commons Attribution - Pas d'Utilisation Commerciale - Pas de Modification 4.0 International. 


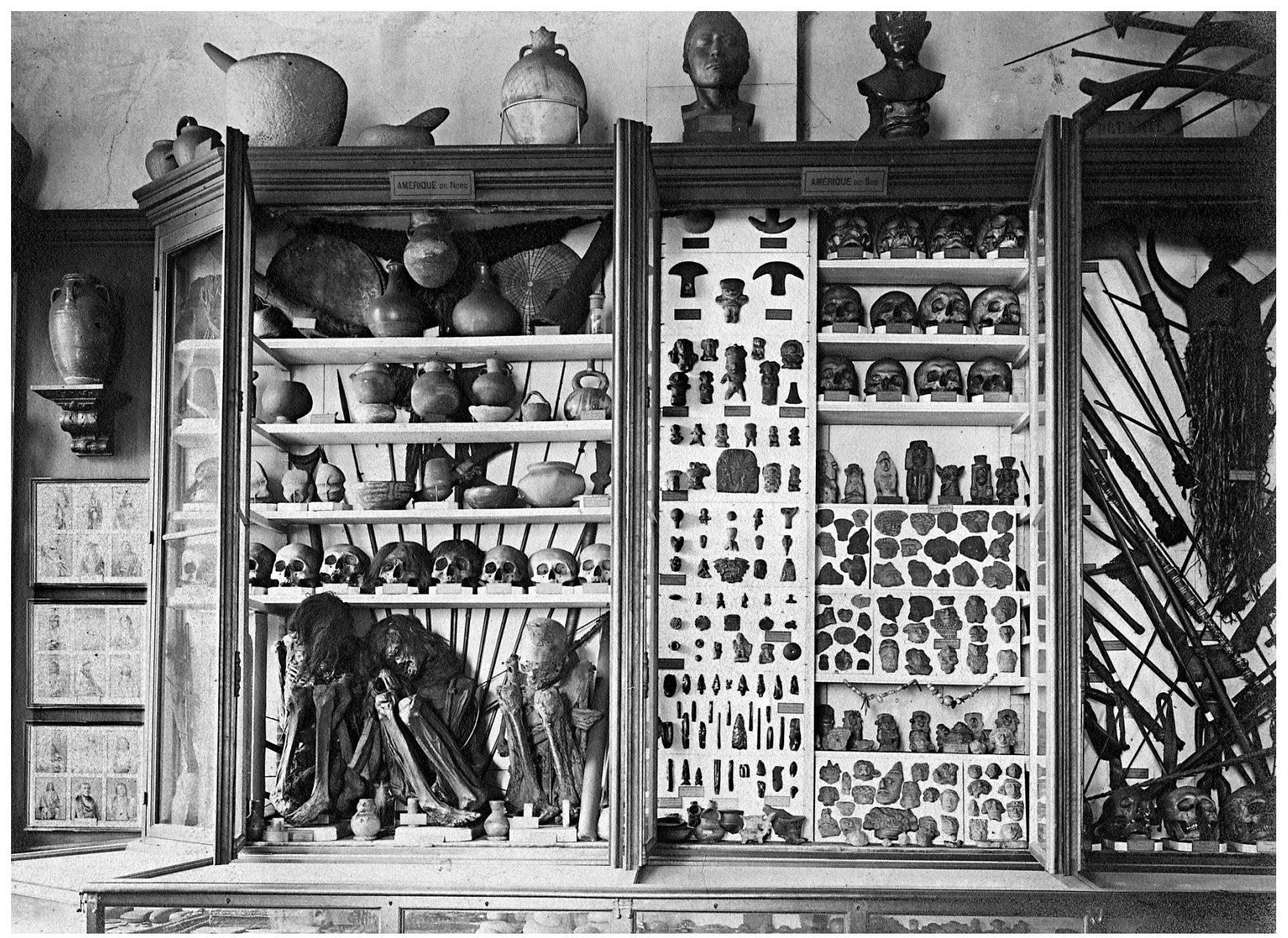

Fig. 1. Vitrines Amérique du Nord et Amérique du Sud du Muséum d'histoire naturelle de Lyon au Palais Saint-Pierre vers 1900,

musée des Confluences (Lyon, France), cote PH8153.

(c) Photographe anonyme. 
Noëlle Timbart

Hélène Guichard

Alain Froment

\section{Un sujet inhabituel pour la revue Technè}

\section{Introduction}

C'est un fait, la mort est présente au musée. Les morts plutôt. Et sous de multiples formes. Depuis les cabinets de curiosité fondateurs jusqu'aux collections publiques du XXI ${ }^{\mathrm{e}}$ siècle, loin d'être dérobées au regard des vivants comme il sied généralement aux cadavres, des dépouilles humaines sont exposées (fig. 1), contemplées par les visiteurs, étudiées par les professionnels. Leur intégration patrimoniale - en France les restes humains patrimonialisés sont inaliénables et imprescriptibles, au même titre que tout objet inscrit à l'inventaire d'un musée public - contribue à la fois à leur sanctuarisation et à leur réification. Comme les ouvres d'art ou les objets archéologiques, elles ont vocation à être conservées, restaurées, étudiées, exposées et transmises aux générations futures.

Momies égyptiennes ou sud-américaines, vestiges osseux archéologiques, préparations anatomiques, reliques mémorielles de célébrités ou humbles restes anonymes, le corps des morts - intègre ou fragmentaire, volontairement ou fortuitement préservé - est entré au musée par de multiples voies. Les fouilles archéologiques, la collecte ethnographique, l'enseignement médical ou l'étude anthropologique en furent les pourvoyeurs les plus coutumiers. Si bien que ces collections marginales, aux aspects et aux origines multiples, constituent aujourd'hui un précieux recueil de la diversité humaine, à travers le temps et l'espace : l'Homme et ses pratiques, sociales, religieuses, médicales, se livrent et se lisent à livre ouvert dans ces véritables archives de l'humanité que constituent les restes humains patrimonialisés, préservés et accessibles. Ils composent en effet une documentation riche d'informations sur la grande famille humaine et le témoignage qu'ils représentent est une source inestimable pour quiconque veut connaître et dévoiler le passé, notre passé, individuel et collectif.

Mais ces « archives » d'un genre particulier exigent d'être traitées avec le respect que toute civilisation humaine réserve aux morts et cette situation atypique, associée au paradoxe que constitue le fait de conserver ce qui par nature est voué à la destruction et d'exposer ce qui est habituellement dissimulé aux vivants, suscite de nombreuses réflexions dans nos institutions patrimoniales du XXI ${ }^{\mathrm{e}}$ siècle. La communauté professionnelle s'attache depuis quelques années à débattre de ces questions ${ }^{1}$ et à proposer des solutions, éthiques ${ }^{2}$ et techniques, relayées (inégalement selon les pays) par les pouvoirs publics ${ }^{3}$. Ce numéro thématique de Technèse propose donc de livrer un état de la question à travers un large éventail de sujets connexes, tant juridiques qu'historiques, scientifiques et techniques, abordant les problématiques de statut, d'étude et d'investigation scientifique, d'exposition et de conservation-restauration, essentiellement en France, mais aussi à l'étranger.

\section{Utilité et particularismes de ces collections}

Les institutions muséales qui conservent ce type de collections et sont mises à l'honneur dans ce numéro peuvent - et doivent - jouer un rôle capital dans la compréhension de l'évolution de l'Homme. Or, l'étude des restes humains a beaucoup évolué. L'anthropologie biologique, qui s'attache à décrire et expliquer l'évolution humaine, est fille de la médecine et, comme elle, s'intéresse à l'anatomie, non pour diagnostiquer des pathologies, mais pour décrire les variations qui constituent la diversité humaine. Cette filiation n'est nulle part plus apparente qu'au Muséum national d'histoire naturelle, détenteur de la plus large collection de restes humains de France, où la chaire d'anthropologie est un avatar direct de la chaire d'anatomie. La médecine légale partage beaucoup de cet héritage puisqu'elle compte parmi ses objectifs la recherche des causes de la mort, mais souvent aussi l'identification de corps d'inconnus, basée sur l'estimation du sexe, de la taille, de l'âge et de l'origine géographique. Même si, dans ce domaine comme ailleurs, la génétique prend le dessus, l'intérêt de la morphologie demeure. Aussi les musées d'anthropologie comme ceux de médecine se sont-ils attachés à collecter des

Noëlle Timbart, conservateur du patrimoine chargé des Antiquités égyptiennes et orientales et des restes humains, département Restauration, C2RMF (noelle.timbart@culture.gouv.fr). Hélène Guichard, conservateur en chef, département des Antiquités égyptiennes, musée du Louvre (helene.guichard@louvre.fr). Alain Froment, directeur de recherche à l'IRD, ancien responsable scientifique des collections d'anthropologie du musée de l'Homme (froment@mnhn.fr). 
ossements ou des pièces naturalisées, et des momies parfois, pour l'enseignement des étudiants et l'instruction du public.

Ce numéro a été conçu pour effectuer la synthèse des questions tant techniques que morales soulevées par ces collections anatomiques, qui tirent leur particularité du fait qu'elles conservent ou mettent en scène nos propres parents, dans l'espace et dans le temps, nos frères et sœurs en humanité, nos «alter-égaux ${ }^{4}$ ». En les contemplant, c'est notre condition de mortels que nous regardons, c'est en fait de notre propre rapport à l'existence, et aux conceptions intellectuelles ou spirituelles qui fondent nos croyances, qu'il s'agit. S'il y a des objets sacrés dans nos idéologies, les plus émouvants, les plus terribles, sont les dépouilles de nos ancêtres. On ne peut donc manipuler ces témoins sans précautions, et les lois de bioéthique ont rappelé l'exigence de respect et de dignité qui leur est due. Mais si la déontologie professionnelle s'applique, ces individus inanimés entrés dans le patrimoine muséal ne sont plus des patients auxquels s'appliquerait le secret médical, ils deviennent des objets de science (fig. 2), et c'est là que le mot « archive » prend tout son sens.

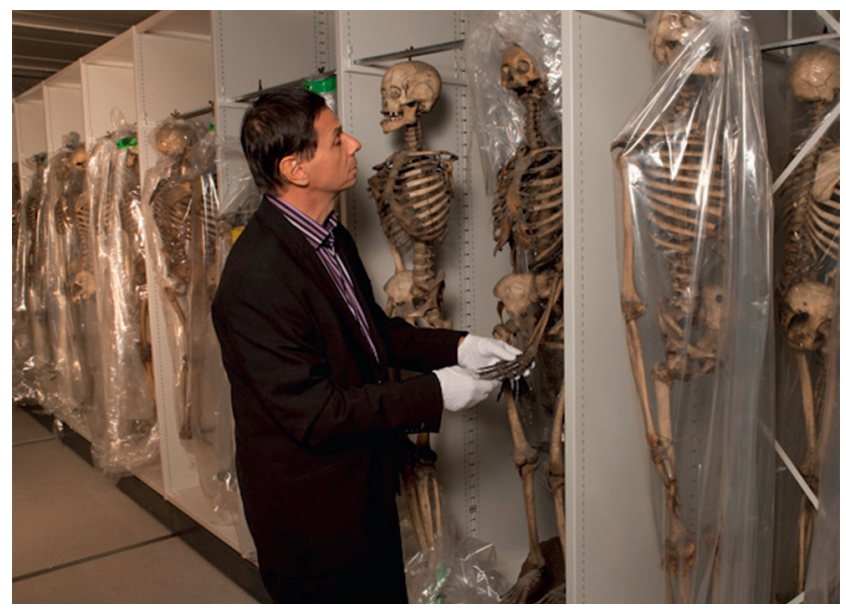

Fig. 2. Étude de la collection de squelettes au musée de l'Homme. (c) P. Goedegheluck.

En effet, de la même façon que ce que nous consommons de licite ou d'illicite va se déposer dans nos cheveux, les événements de notre vie vont sédimenter dans notre squelette, permettant d'écrire, pour chacun d'entre nous, une ostéobiographie, et même une odontobiographie à partir d'une simple dent. Aussi avons-nous passé en revue les techniques de la bio-archéologie, qui consiste à reconstituer le mode de vie, l'alimentation et la pathologie des populations disparues. Des progrès considérables ont été enregistrés tant dans le domaine de l'imagerie, qui génère une iconographie tridimensionnelle et colorée extraordinaire, bien loin des tristes radiographies d'antan, que dans celui des analyses de laboratoire, mobilisant toute la palette de la physique et de la chimie.
Une des surprises majeures de ces dernières décennies a été la découverte d'ADN dans les os anciens ; après des débuts balbutiants, on parvient à présent à des résultats qui dépassent l'imagination, comme le séquençage de génomes complets d'individus vieux de 100000 ans. Cela montre, s'il en était besoin, l'intérêt de conserver dans nos musées ces archives biologiques pour les générations futures, qui pousseront toujours plus loin les méthodes d'investigation. Pourtant, en raison du fort symbole identitaire et religieux que constituent ces restes, notamment pour les peuples victimes de la colonisation, et dont les reliques d'ancêtres ont souvent été récoltées sans leur accord, les demandes de restitution constituent un enjeu de négociation symbolique important, souvent appuyé par les gouvernements issus de cette même colonisation, qui voient là un moyen de transposer sur le plan spirituel un mode de réparation qui évite la restitution des terres et des richesses. Il convient de traiter ces demandes avec diplomatie et clairvoyance, en s'appuyant sur une véritable épistémologie des conditions de collecte et du contexte idéologique contemporain.

\section{Contenu et limites de ce numéro}

Étant donné la multiplicité des restes humains patrimonialisés dans les collections muséales, tous les domaines n'ont pu être abordés, à l'instar des reliques qui ont fait l'objet de plusieurs publications et expositions, ou de l'art contemporain. Ce dernier domaine nous paraissait à la limite de notre problématique car, bien souvent, les restes humains y sont détournés de leur fonction originale, comme L'oisillon de Dieu de Jan Fabre (2000) ou Stèle et crâne néolithique de Raynaud (1985) par exemple. Ils posaient également des questions d'ordre éthique et déontologique supplémentaires auxquelles il aurait été nécessaire de consacrer une partie importante.

De même, nous n'avons pas souhaité présenter de synthèse sur la conservation préventive des restes humains. Plusieurs articles évoquent cette question et mettent en évidence qu'une démarche spécifique doit être adoptée pour chaque cas. Si celle-ci est essentielle pour ces collections fragiles, elle ne leur est néanmoins en rien spécifique car s'appliquent alors les mêmes recommandations que pour toute autre collection contenant des restes organiques. C'est du côté des vitrines de musées que plusieurs projets ont été menés récemment. À l'occasion de rénovations, plusieurs musées ont entrepris des opérations de conservation-restauration de leurs collections, impliquant la mise en place in fine de nouvelles vitrines. Le C2RMF a pu accompagner plusieurs de ces projets. Il mène actuellement, par l'intermédiaire du département Conservation préventive, des études sur les vitrines qui devraient permettre, à partir des données obtenues, d'améliorer les conditions d'exposition de ces collections extrêmement fragiles.

En revanche, nous avons choisi de confronter les expériences françaises et étrangères en présentant des catégories 
d'objets peu ou pas représentés dans les collections nationales, telle que la question des Natifs américains ou des corps des tourbières. Nous avons en outre voulu illustrer la variété des collections de restes humains en présentant des cas d'étude relevant de diverses civilisations.

Nous avons choisi de concentrer notre propos sur quatre grands domaines, structurant ce numéro en quatre parties : la première porte sur les aspects juridiques et légaux qui entourent ces collections ; la deuxième a trait à l'histoire des collections et à la muséographie ; la troisième aux études et recherches qui sont menées dans ce domaine tandis que la dernière concerne les approches de conservation-restauration.

Nous espérons que cet opus thématique permettra de donner aux lecteurs un aperçu des problématiques que posent les collections de restes humains, mais également de la richesse de ces collections trop souvent remisées et considérées comme d'un intérêt mineur. Cet objectif ne saurait être atteint sans un travail interdisciplinaire, que ce soit dans le domaine de la recherche historique, scientifique et technique que de la conservation.
Notes

1. En France, colloques et travaux académiques ont fait l'objet de publications désormais incontournables. Le lecteur se reportera par exemple aux actes du colloque organisé au musée du quai Branly en février 2008 (Des collections anatomiques aux objets de culte : conservation et exposition des restes humains dans les musées) ou à l'ouvrage de Laure Cadot, En chair et en os : le cadavre au musée. Valeurs, statuts et enjeux de la conservation des dépouilles humaines patrimonialisées, École du Louvre/RMN, Paris, 2009.

2. Voir par exemple Jean-Claude Ameisen, Pierre Le Coz (rap.), Avis $n^{\circ} 111$ : avis sur les problèmes éthiques posés par l'utilisation des cadavres à des fins de conservation ou d'exposition muséale, Paris, Comité consultatif national d'éthique pour les sciences de la vie et de la santé, 7 janvier 2010, 15 p

3. Pour un aperçu de la question du statut des restes humains patrimonialisés et du débat juridique qui les entoure :
Marie Cornu, « La condition juridique des restes humains, l'évolution récente du contexte légal ", Les cadavres de nos musées sont-ils exquis? Les "restes" humains dans les musées, Musées et Collections publiques de France, n 259 (2010-2).

4. Néologisme pressenti pour une prochaine exposition sur le racisme au musée de l'Homme, et déjà utilisé par plusieurs structures ou associations. 